\title{
Energy Harvesting in Microscale with Cavitating Flows
}

\author{
Morteza Ghorbani, ${ }^{\dagger, \ddagger, \S, \perp}$ Ali Mohammadi, ${ }^{\ddagger}, \perp$ Ahmad Reza Motezakker, ${ }^{\ddagger}, \perp$ Luis Guillermo Villanueva, ${ }^{\S}$ \\ Yusuf Leblebici, ${ }^{\S}$ and Ali Koşar*, ${ }^{\ddagger}, \S, \|, \perp$ (1) \\ ${ }^{\dagger}$ Sabanci University Nanotechnology Research and Application Center, 34956 Tuzla, Istanbul, Turkey \\ ${ }^{*}$ Mechatronics Engineering Program, Faculty of Engineering and Natural Science, Sabanci University, 34956 Tuzla, Istanbul, Turkey \\ ${ }^{\S}$ Department of Mechanical Engineering and "Institute of Electrical Engineering, École Polytechnique Fédérale de Lausanne, \\ Lausanne CH-1015, Switzerland \\ ${ }^{\perp}$ Center of Excellence for Functional Surfaces and Interfaces for Nano-Diagnostics (EFSUN), Sabanci University, Orhanli, 34956 \\ Tuzla, Istanbul, Turkey
}

\section{Supporting Information}

ABSTRACT: Energy harvesting from thermal energy has been widely exploited to achieve energy savings and clean technologies. In this research, a new cost-effective and environment-friendly solution is proposed for the growing individual energy needs thanks to the energy application of cavitating flows. With the aid of cavitating jet flows from microchannel configurations of different sizes, it is shown that significant temperature rise (as high as $5.7^{\circ} \mathrm{C}$ ) can be obtained on the surface of the thin plate. The obtained heat energy could be integrated to a thermoelectric power generator, which can be used as a power resource for consumer devices, such as cell phones and laptops. To explore the difference in the temperature rise with different microtube diameters, namely,

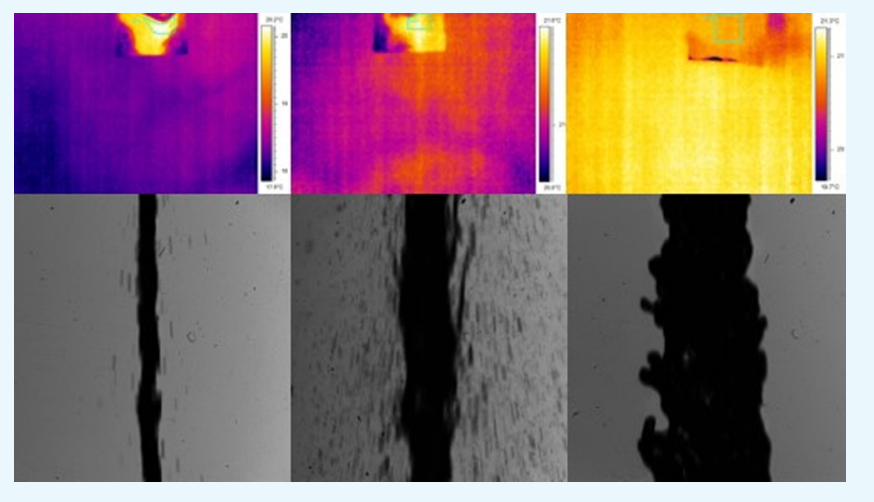
$152,256,504$, and $762 \mu \mathrm{m}$, and also with different upstream pressures of 10,20,40, and 60 bar, the cavitation flow patterns are captured and analyzed using an advanced high-speed visualization system. The analysis of the captured data showed that different flow patterns exist for different diameters of the microtubes, including a pattern shift from micro- to macroscale, which accompanied the pattern of temporal results very well.

\section{INTRODUCTION}

Hydrodynamic cavitation is a progressive cycle of vaporization, bubble generation, and bubble implosion and is one of the major phase change phenomena. The effects of hydrodynamic cavitation have been examined by many researchers and are included in the literature. ${ }^{1-5}$ It occurs when the local static pressure is reduced to a critical value. Small bubbles are generated in small flow-restrictive elements, which trigger a sudden reduction in the local pressure of the flows and cause cavitation inception. The collapse of the cavitation bubbles downstream of these elements leads to a high energy release, thereby generating highly localized, large-amplitude shock waves and high-velocity jets. ${ }^{6,7}$ Thus, hot spots of over 1000 $\mathrm{K}$ and high-speed microjets exceeding $300 \mathrm{~m} / \mathrm{s}$ have been achieved as a result of the collapse of the cavitation bubbles. ${ }^{8,9}$ Although jet impingement gives rise to cooling on an exposed surface and is considered an effective cooling method, ${ }^{10-12}$ a rise in the surface temperature can be instead attained in the presence of intense bubbly cavitating flows downstream of the flow-restrictive elements. Our recent study illustrates that many cavitation bubbles were generated in channels with inner diameters of 256 and $504 \mu \mathrm{m}$ and filled more space inside the channel compared to the conventional channels at higher upstream pressures, ${ }^{13}$ which increased the possibility of the occurrence of collapse process at the outlet of the microchannels. These findings imply that the proposed method in this study is feasible. Multiple cavitation bubbles in the proximity of a surface constitute a heat source with uniform temperature distribution, which can be integrated to a thermoelectric generator to power daily-used devices. This effect constitutes the motivation of the present study.

Small bubbles have many potential applications in the pharmaceutical, food, and chemical industries. In the field of medicine, nano-/microbubbles are expected to be applicable in areas such as the development of ultrasound contrast agents, ${ }^{14}$ targeted drug delivery, tumor destruction, and tumor imaging. ${ }^{15}$ In the food industry, the density and texture of gel- and creambased foods could be controlled by the uniform and fine dispersion of gases into these materials. ${ }^{16}$

Two types of studies were considered in the literature on the field of the proposed system. On the one hand, spraying is used as a technology to provide cooling heat transfer on the exposed

Received: August 17, 2017

Accepted: October 5, 2017

Published: October 18, 2017 


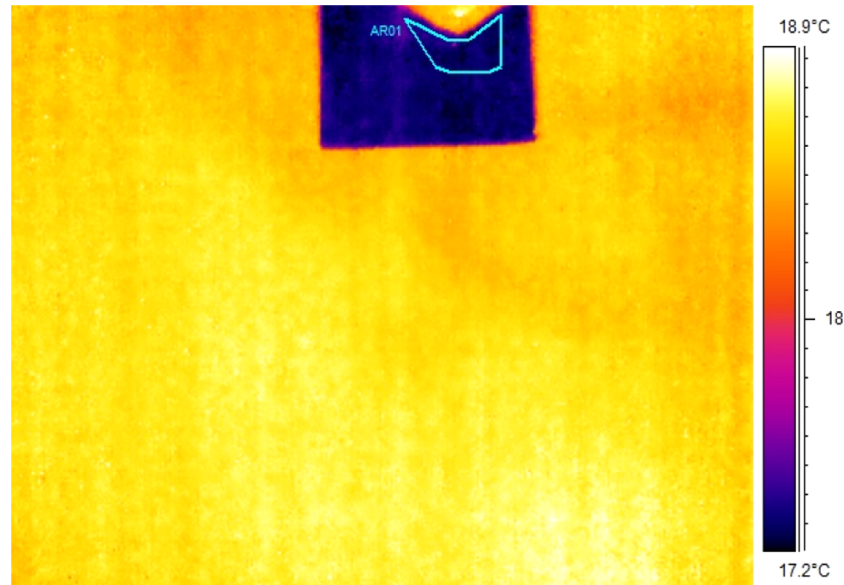

(a)
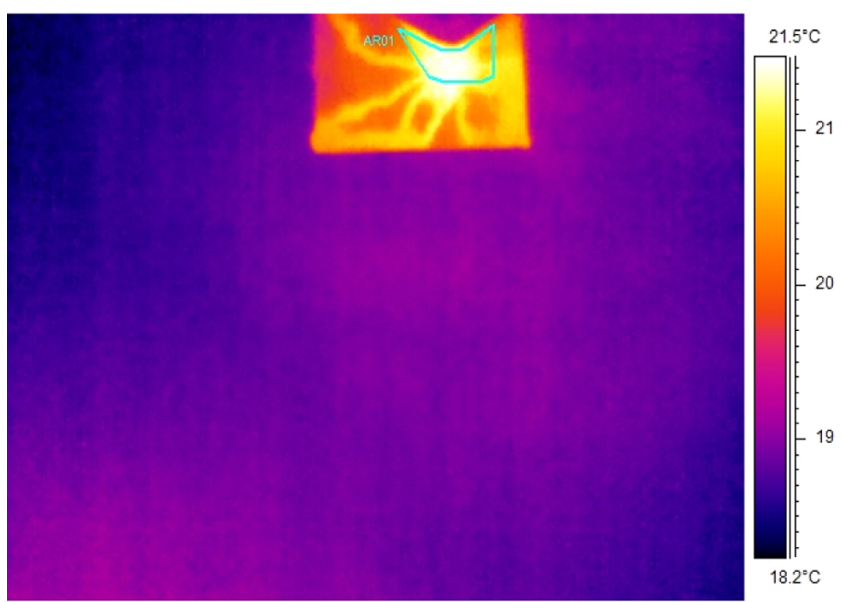

(c)

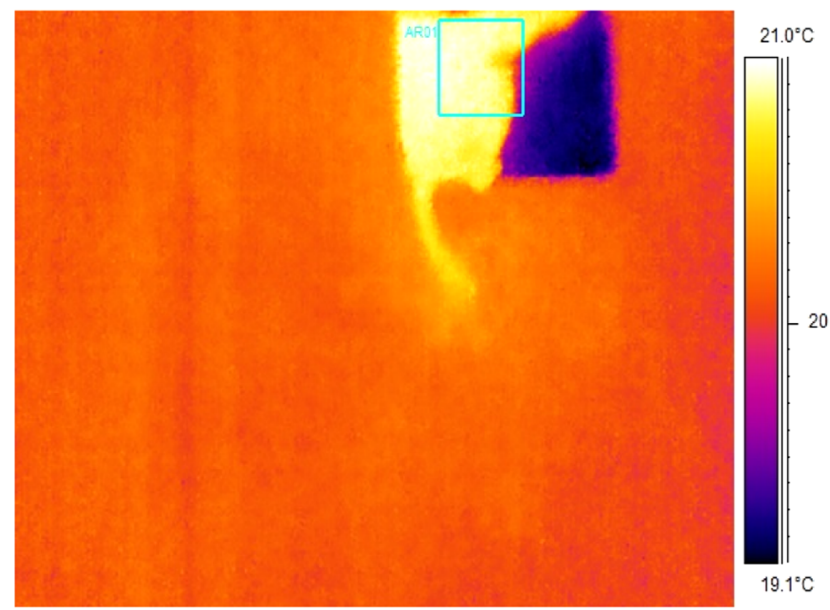

(e)

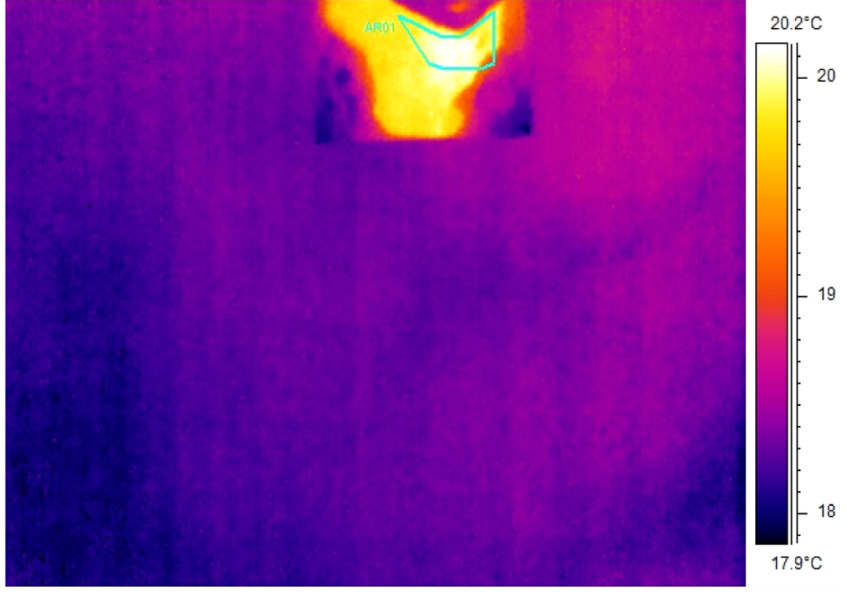

(b)

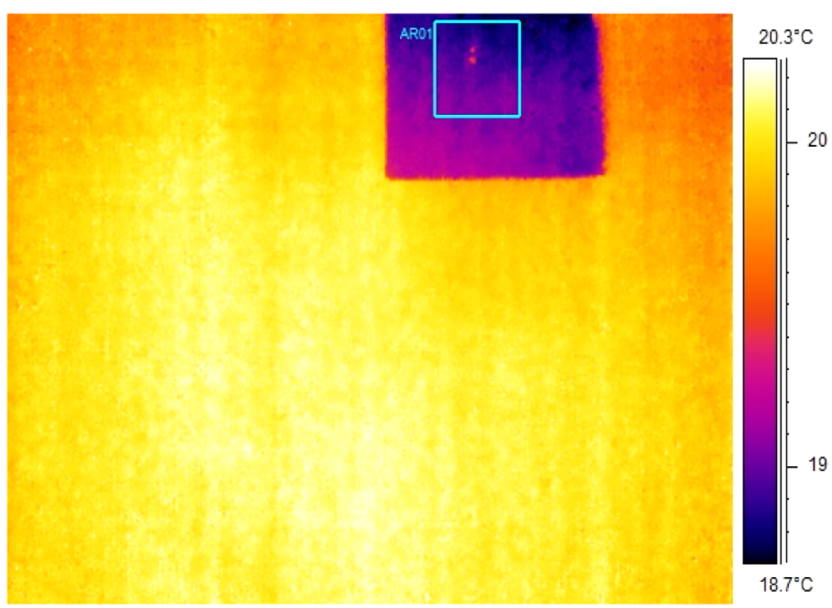

(d)

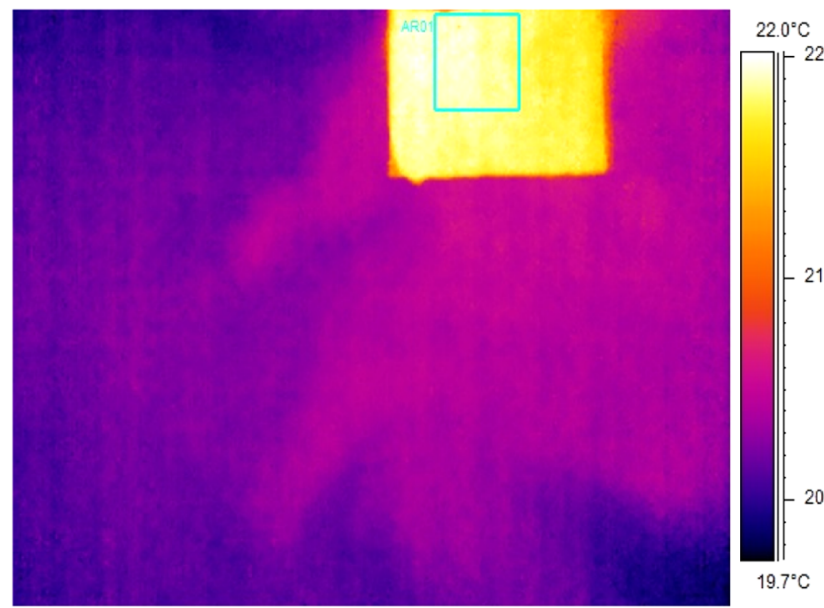

(f)

Figure 1. Thermal contours captured by a thermal camera on the solid surface for different cases: (a) without spray exposure (152 $\mu \mathrm{m}),(\mathrm{b})$ just after the exposure $(152 \mu \mathrm{m}),(\mathrm{c}) 30 \mathrm{~s}$ after the exposure $(152 \mu \mathrm{m}),(\mathrm{d})$ without spray exposure $(504 \mu \mathrm{m})$, (e) just after the exposure $(504 \mu \mathrm{m})$, and (f) 30 $\mathrm{s}$ after the exposure $(504 \mu \mathrm{m})$. The selected box on the surfaces is the location where the emerging cavitation jet hits the surface. This approach helps to measure the mean temperature, where the most direct effect of the spray jet impingement occurs. AR01 represents the most relative area that the jet influences on the plate. It was tried to consider the same area of $1 \times 1 \mathrm{~mm}^{2}$ for all cases. However, as the area which was directly impacted by the jet from the tube with the inner diameter of $152 \mu \mathrm{m}$ was less than $1 \mathrm{~mm}^{2}$, a smaller area was introduced for this microtube. 
(a)

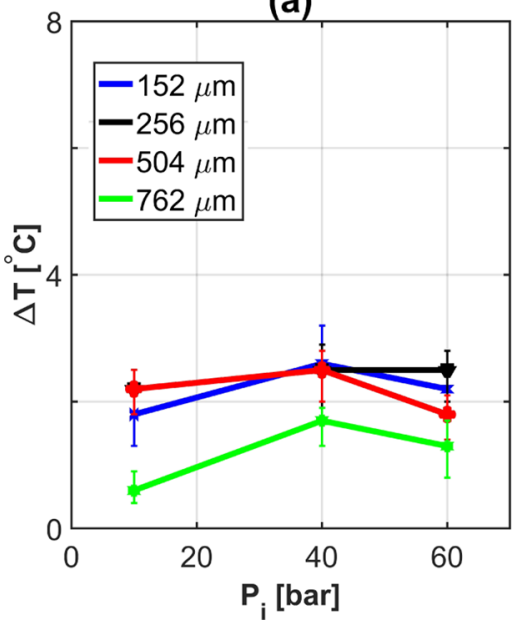

(b)

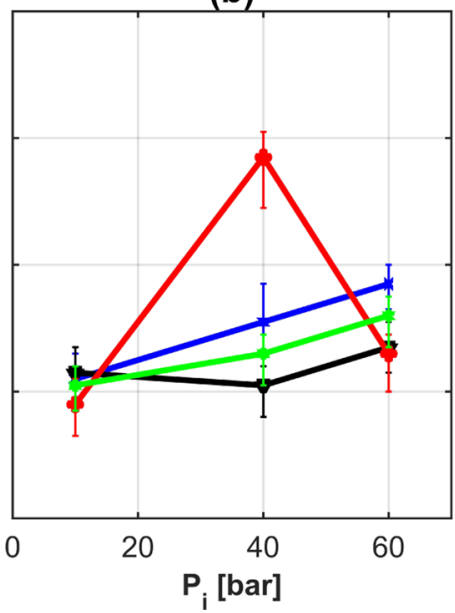

(c)

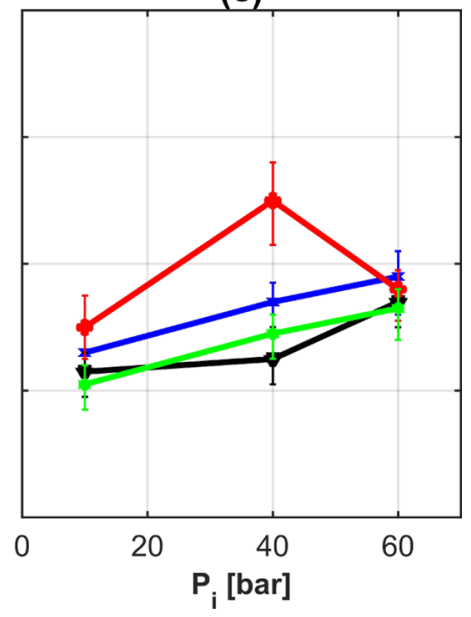

Figure 2. Surface temperature rise as a function of upstream pressure: (a) just after the exposure to the surface, (b) $30 \mathrm{~s}$ after the exposure, and (c) $120 \mathrm{~s}$ after the exposure. The thermal contours are analyzed at three time steps to monitor and confirm the stability and steadiness of the temperature rise, which is needed to ensure reliable power harvesting. The room temperatures for the selected experiment sets were measured as $17.3,19.3,19$, and $19.6^{\circ} \mathrm{C}$ for the channel configurations with diameters of $152,256,504$, and $762 \mu \mathrm{m}$, respectively. Overall, the extracted results shown in this figure depict a consistent temperature rise between 0.8 and $5.7^{\circ} \mathrm{C}$ for all of the channels at different upstream pressures.

targets. In this regard, due to the high heat flux removal, high heat dissipation rate, and low superheat, spray cooling has been used in different areas of industry. ${ }^{17}$

On the other hand, utilization of the thermal gradient generated during the collapse of the ultrasound cavitation bubble is considered in a broad range in the literature. The gas inside the bubble has high temperature and pressure during the collapse point. ${ }^{18}$ Moreover, the cavitation impact of the power loss and heat dissipation due to the bubble generation and noise generation in the hydraulic machinery has been considered in the literature recently. ${ }^{19}$ It was predicted that the cavitation phenomenon would have a strong potential for heat generation from an energetic point of view.

In this study, compared to the studies available in the literature, we propose a novel approach in microscale utilizing the energy produced during the interaction of the spray affected by the hydrodynamic cavitating flow and a thin aluminum plate. With a decrease in size, cavitation effects become significant. It is clearly shown that with the aid of hydrodynamic cavitation generated inside the micro-/minichannels in addition to the optimization of the distance between the tip of the microchannel configuration and the solid surface, surface temperatures can be increased up to $5{ }^{\circ} \mathrm{C}$ under the conditions of this study. The temperature rise on the surfaces near the collapsing small bubbles was exploited for energy harvesting in small scale in such a way that miniature, cost-effective, and environmentfriendly energy-harvesting devices can be developed. Such devices will not require any external power and moving parts in contrast to common energy-harvesting devices, such as those involving piezoelectric materials ${ }^{20,21}$ and microengines. ${ }^{22,23}$ In this study, the capability of the hydrodynamic cavitation in microscale as a strong alternative to the ultrasound cavitation is presented in terms of clean and cheap energy harvesting, which has not been considered in the literature to the best of our knowledge.

\section{RESULTS AND DISCUSSION}

The results show a temperature rise in all of the cases. In Figure 1 , the contours on the left side of each row indicate the temperature profile before jet impingement. The subsequent contours show the temperature variation after approximately 1 (named as 0 ) and $30 \mathrm{~s}$ surface exposure to the cavitating jet. The reduced data obtained with the FLIR software (FLIR Systems) from the analysis of the thermal contours of all cases are presented in Figure 2. The detailed data for all cases are shown in Supporting Information.

Figure 2a shows that there is a temperature rise for all of the channels, starting from upstream pressures of 10-60 bar, just after the emerging cavitating jet contacts the surface. The largest temperature increase is seen at an upstream pressure of 40 bar. Under this upstream condition, the largest temperature rise is $2.6{ }^{\circ} \mathrm{C}$ in the microchannel configuration with diameter of $152 \mu \mathrm{m}$. A temperature rise of $2.5{ }^{\circ} \mathrm{C}$ is seen in the microchannel configurations with diameters of 256 and $504 \mu \mathrm{m}$ at the same upstream pressure. The microchannel configuration with diameter of $762 \mu \mathrm{m}$ has an increase of $1.7^{\circ} \mathrm{C}$, which is the lowest recorded temperature rise at this pressure. For all of the channels, with an increase in the upstream pressure from 40 to 60 bar, the surface temperature either remains constant or moderately decreases. The optimal pressure depends on the diameter in a way that the optimal pressure for $504 \mu \mathrm{m}$ is 40 bar, but the optimal pressure for 152,256 , and $762 \mu \mathrm{m}$ is 60 bar.

Similarly, Figure $2 \mathrm{~b}$ illustrates, for all of the cases, the reduced data corresponding to $30 \mathrm{~s}$ after the exposure of the solid body to the cavitating flows. As can be seen, the temperature increases with time. Further, as time elapses, it can be seen that the rise in surface temperature becomes saturated, which also implies an optimum rise in temperature for the studied cases. The comparison of Figure $2 b$ with Figure $2 c$ reveals that temperature does not significantly change between the time steps of 30 and $120 \mathrm{~s}$ after exposure, thus implying steady-state conditions after a time lapse.

In the second time step, the highest temperate rise is recorded as $5.7^{\circ} \mathrm{C}$ in the microchannel with inner diameter of $504 \mu \mathrm{m}$ at an upstream pressure of 40 bar. Because this temperature rise is significantly higher than those for the other micro-/minichannel configurations, additional experiments were performed to ensure the repeatability of the results. This finding originated from the corresponding flow patterns 


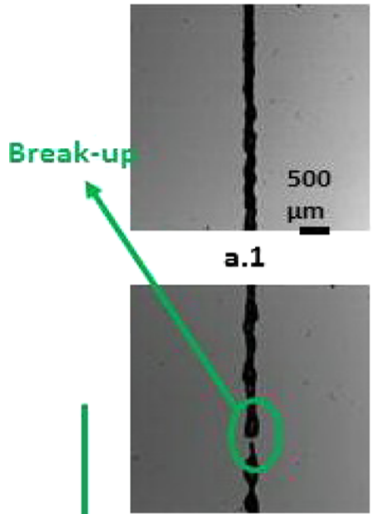

b.1

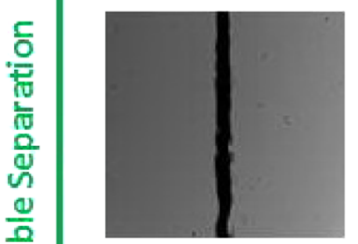

c.1

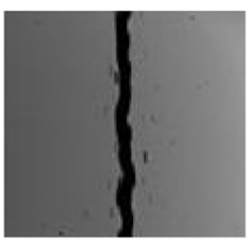

d. 1

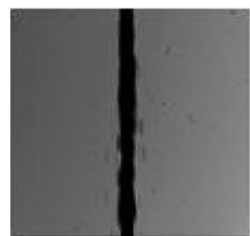

e.1

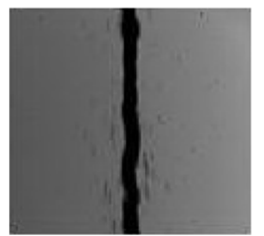

f.1

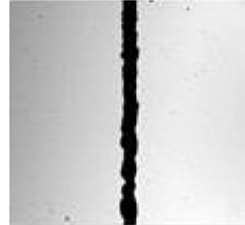

a. 2

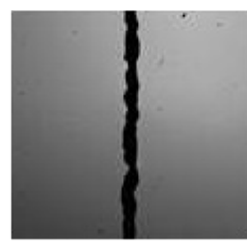

b. 2

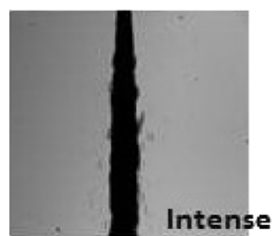

c.2 Cloud

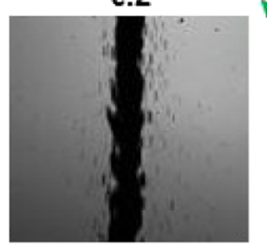

d. 2

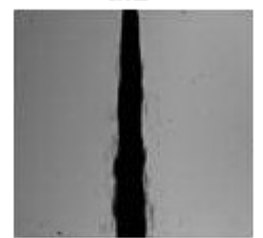

e. 2

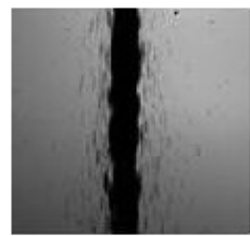

f. 2

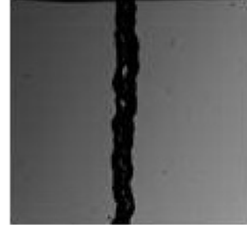

a.3

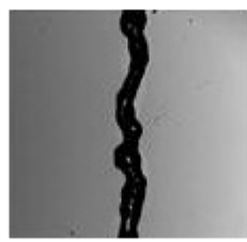

b. 3

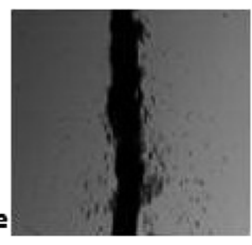

c. 3

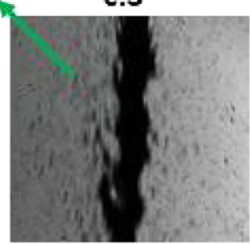

d. 3

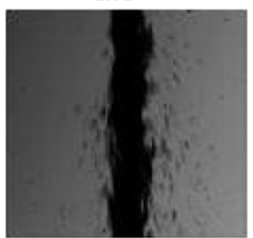

e. 3

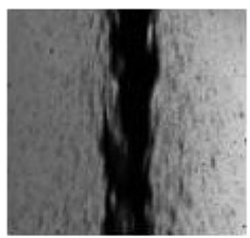

f. 3

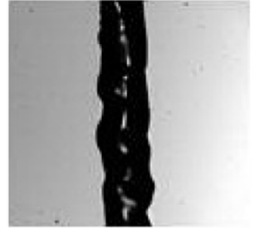

a.4

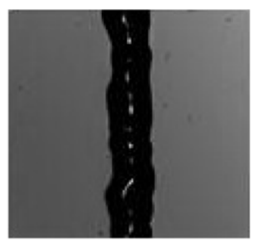

b. 4

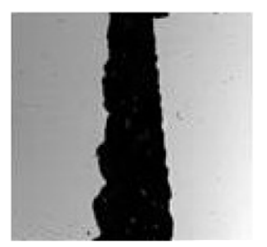

c. 4

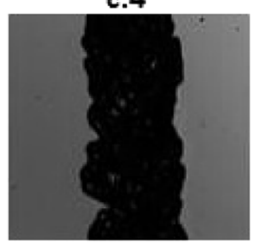

d. 4
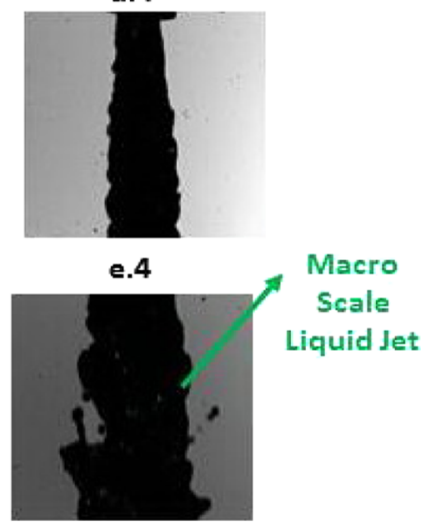

f. 4

Figure 3. Flow patterns at the outlet of the micro-/minichannels for different upstream pressures at the first and last segments of the spray structure. The emerging spray has a conical shape in almost all of the channels, except that with diameter of $762 \mu \mathrm{m}$. The flow patterns significantly change as the diameter of the channel shifts from 504 to $762 \mu \mathrm{m}$, which implies a difference between micro- and macroscale cavitation phenomena. $\left(\right.$ a.1) $D_{\mathrm{i}}=$ $152 \mu \mathrm{m}, P_{\mathrm{i}}=10$ bar, segment 1 ; (a.2) $D_{\mathrm{i}}=256 \mu \mathrm{m}, P_{\mathrm{i}}=10 \mathrm{bar}$, segment 1 ; (a.3) $D_{\mathrm{i}}=504 \mu \mathrm{m}, P_{\mathrm{i}}=10$ bar, segment $1 ;\left(\right.$ a.4) $D_{\mathrm{i}}=762 \mu \mathrm{m}, P_{\mathrm{i}}=10$ bar, segment 1; (b.1) $D_{\mathrm{i}}=152 \mu \mathrm{m}, P_{\mathrm{i}}=10$ bar, segment 6; (b.2) $D_{\mathrm{i}}=256 \mu \mathrm{m}, P_{\mathrm{i}}=10$ bar, segment 6; (b.3) $D_{\mathrm{i}}=504 \mu \mathrm{m}, P_{\mathrm{i}}=10$ bar, segment 6; (b.4) $D_{\mathrm{i}}$ $=762 \mu \mathrm{m}, P_{\mathrm{i}}=10 \mathrm{bar}$, segment 6 ; (c.1) $D_{\mathrm{i}}=152 \mu \mathrm{m}, P_{\mathrm{i}}=40$ bar, segment 1 ; (c.2) $D_{\mathrm{i}}=256 \mu \mathrm{m}, P_{\mathrm{i}}=40$ bar, segment 1 ; (c.3) $D_{\mathrm{i}}=504 \mu \mathrm{m}, P_{\mathrm{i}}=40$ bar, segment 1; (c.4) $D_{\mathrm{i}}=762 \mu \mathrm{m}, P_{\mathrm{i}}=40$ bar, segment 1; (d.1) $D_{\mathrm{i}}=152 \mu \mathrm{m}, P_{\mathrm{i}}=40$ bar, segment 6; (d.2) $D_{\mathrm{i}}=256 \mu \mathrm{m}, P_{\mathrm{i}}=40$ bar, segment 6; (d.3) $D_{\mathrm{i}}=504 \mu \mathrm{m}, P_{\mathrm{i}}=40$ bar, segment 6; (d.4) $D_{\mathrm{i}}=762 \mu \mathrm{m}, P_{\mathrm{i}}=40$ bar, segment 6; (e.1) $D_{\mathrm{i}}=152 \mu \mathrm{m}, P_{\mathrm{i}}=60$ bar, segment 1 ; (e.2) $D_{\mathrm{i}}=256 \mu \mathrm{m}$, $P_{\mathrm{i}}=60$ bar, segment 1 ; (e.3) $D_{\mathrm{i}}=504 \mu \mathrm{m}, P_{\mathrm{i}}=60$ bar, segment 1 ; (e.4) $D_{\mathrm{i}}=762 \mu \mathrm{m}, P_{\mathrm{i}}=60$ bar, segment 1 ; (f.1) $D_{\mathrm{i}}=152 \mu \mathrm{m}, P_{\mathrm{i}}=60$ bar, segment 6; (f.2) $D_{\mathrm{i}}=256 \mu \mathrm{m}, P_{\mathrm{i}}=60 \mathrm{bar}$, segment 6; (f.3) $D_{\mathrm{i}}=504 \mu \mathrm{m}, P_{\mathrm{i}}=60 \mathrm{bar}$, segment 6; (f.4) $D_{\mathrm{i}}=762 \mu \mathrm{m}, P_{\mathrm{i}}=60$ bar, segment 6 .

illustrated in Figure 3, especially when considering the flow patterns of the micro-/minichannel configurations with inner diameters of 504 and $762 \mu \mathrm{m}$.

The temperature rise obtained from the largest channel configuration, which corresponds to a tube diameter of $762 \mu \mathrm{m}$, is smaller than that in the other cases just after the spray exposure to the surface. A rise of $3.2{ }^{\circ} \mathrm{C}$ is recorded for this configuration after a $30 \mathrm{~s}$ exposure, and the nearest result is 4.3
${ }^{\circ} \mathrm{C}$, obtained from the configuration with diameter of $152 \mu \mathrm{m}$. The same trend of rise in temperature with time occurs for all of the configurations. The largest temperature rise, of approximately $4{ }^{\circ} \mathrm{C}$, is attained from the microchannel configurations with diameters of 504 and $152 \mu \mathrm{m}$. The experiments were repeated four times for each case, where all showed reasonably similar outcomes. However, for the cases which corresponded to peak points, the experiments were 


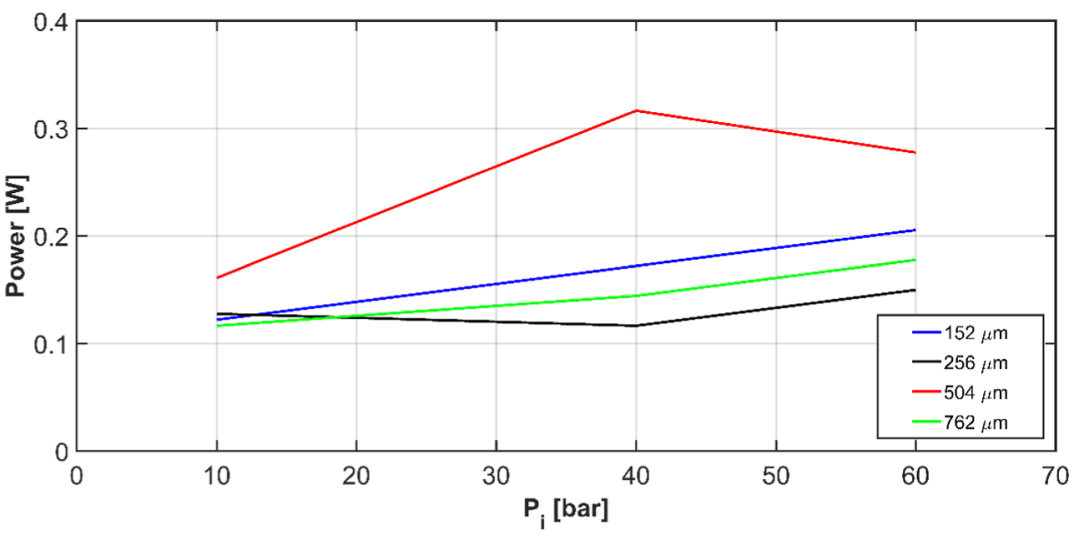

Figure 4. Output power generated as a result of the exposure of the solid surface to the cavitating jets.

repeated two or three times more than usual to ensure the repeatability of the results.

To complement the results of the temperature profiles, the flow patterns are analyzed at the outlet of the channel configurations using a high-speed visualization system and a laser shadow probe. Visualization experiments are performed for all of the configurations at six different consecutive segments throughout the spray, starting from the channel exits. Each segment has a length of $3 \mathrm{~mm}$. As shown in Figure 3 , for most of the segments in the channels with inner diameters of 152 and $256 \mu \mathrm{m}$, the flow structures are similar. With the increase in diameter from 256 to $504 \mu \mathrm{m}$, an intense separation of little droplets from the main jet is observed. This is in agreement with the change in the temperature rise trends mentioned earlier. As diameter increases to $762 \mu \mathrm{m}$, the flow pattern becomes different and a bigger jet comes out of the channel. This change in the flow pattern is accompanied by a lower temperature rise. With an upstream pressure of $10 \mathrm{bar}$ (Figure 3a.1-a.4 and 3b.1-b.4), the flow pattern changes slightly by increasing the channel diameter. However, with the upstream pressure of 40 and $60 \mathrm{bar}$, the droplet/bubble separation from the main flow gets more intense along with the spray cloud, especially in the channel with inner diameter of $504 \mu \mathrm{m}$. The transition from micro- to macroscale cavitating flow is more apparent for the channels with inner diameters of $504-762 \mu \mathrm{m}$ at higher upstream pressures. The detailed data for all cases are shown in Supporting Information.

The spray at the outlet section of the channels experiences decomposition along its length, which leads to increasing effects of disintegrated droplets and bubbles from the cavitating jet, during the primary and secondary breakups. The primary breakup leads to the generation of big droplets/bubbles, which causes a dense region close to the orifice. The next stage in the spray structure is a secondary breakup, where smaller droplets/ bubbles form from the big ones. The secondary breakup occurs due to the resisting aerodynamic forces from the surrounding gas acting on the droplets/bubbles. This resisting force reduces the droplet velocity. Furthermore, at the tip of the spray, droplets tolerate much higher drag forces. Therefore, at this location, droplets consistently change to new ones and the spray length increases. Hence, additional and faster droplets/ bubbles interact with the solid body as time passes.

The intensity of cavitation is characterized via the cavitation number, which is expressed as: $\sigma=\left(P_{\text {ref }}-P_{\mathrm{V}}\right) / 0.5 \rho V_{\text {ref }}^{2}$, where $P_{\text {ref }}$ is the reference pressure, which is the upstream pressure in this study, $P_{\mathrm{V}}$ is the vapor saturation pressure (vapor saturation pressure for the working fluid is approximately $3500 \mathrm{~Pa}$ ), and $V_{\text {ref }}$ is the reference velocity, which is the mean velocity in the channel and measured using the flow rate during the flow injection. Accordingly, the cavitation numbers for the channels with diameters of 152 and $762 \mu \mathrm{m}$ are 0.078 and 0.52 , respectively, for the upstream pressure of 40 bar. Thus, cavitation is more intense in channels with smaller diameters. There is a similar trend for the cavitation number at the other injection pressures.

The images in the first row of Figure 3 show the first segments at the upstream pressure of $10 \mathrm{bar}$. At this pressure, the cavitation effects are not intense for any of the channels, which is also in agreement with the previous studies. ${ }^{13,24}$ The flow pattern at this pressure and segment is mainly a liquid jet. However, in the last segment, different flow patterns exist. For the channel with diameter of $152 \mu \mathrm{m}$ and for the channel with diameters of 256 and $504 \mu \mathrm{m}$, droplet separation and large perturbations, respectively, are observed in the last segment. Large and small perturbations are also observed in the channels with diameters of 256 and $504 \mu \mathrm{m}$, which tend to form a spray jet under the minimal energy condition.

In the third and fourth rows of Figure 3, the upstream pressure increases to $40 \mathrm{bar}$, and in the fourth and fifth rows of Figure 3, the upstream pressure increases to 60 bar. It can be seen in the fourth row (which is the sixth section of different microtubes) that an intense breakup pattern exists for microtubes with inner diameters of 152,256 , and $504 \mu \mathrm{m}$. As the upstream pressure increases to $60 \mathrm{bar}$, this intense breakup pattern becomes prevalent in all segments of the channels with diameters of 152,256 , and $504 \mu \mathrm{m}$, which is accompanied by droplet/bubble separations, suggesting a cloudy spray under the effect of cavitation. The effect of this spray cloud becomes more dominant with increasing temperature at higher injection pressures. However, entirely different patterns can be seen for all segments of the channel with diameter of $762 \mu \mathrm{m}$. The flow pattern resembles that of the liquid jet in macro-/conventionalscale sprays, which is shown in the literature. ${ }^{25}$ Moreover, the cavitation numbers for the channels with diameters of 504 and $762 \mu \mathrm{m}$ are 0.39 and 0.52 , respectively, for the upstream pressure of $40 \mathrm{bar}$. According to the observations and trends in temperature rise presented above, it can be concluded that there is a flow structure change because of the shift from mini-/ macroscale to microscale cavitation, concerning the channels with diameters between 504 and $762 \mu \mathrm{m}$.

According to the results, cavitating flows are more intense for the smallest channel configuration. However, those cavitating 
flows hit a smaller surface area, where the rise in temperature contributes less to the average temperature rise. Although this contribution is greater for larger channel configurations, the cavitation intensity is smaller. The interplay between exposed area and cavitation intensity results in an optimal channel configuration for the average temperature rise and consequent energy harvesting.

The temperature rise with small-scale cavitating flows can be exploited for energy harvesting using thermoelectric generators. According to this study, the obtained power is in the range of the maximum power gained from a thermal harvester, $P_{\mathrm{Max}}=$ $(S \Delta T)^{2} / 4 R$, where $S$ and $R$ are the Seebeck coefficient and resistance of aluminum, respectively. Even taking into account that the energy conversion efficiency of existing thermoelectric elements can be far from ideal (especially at lower voltage values), it can be expected that a temperature difference of $2-3$ ${ }^{\circ} \mathrm{C}$ would result in an output power of around $300 \mathrm{~mW},{ }^{26}$ which can power devices such as radio frequency receivers/ transmitters, light-emitting diodes (LED), and miniature sensors.

Figure 4 displays the output power for the time step of $30 \mathrm{~s}$. It can be seen that the highest power is obtained from the channel with diameter of $504 \mu \mathrm{m}$, which is in agreement with Figure $2 \mathrm{~b}$. At the upstream pressure of $40 \mathrm{bar}$, the maximum reclaimable power is $0.316 \mathrm{~W}$ for this channel. This configuration is followed by the channel with diameter of 152 $\mu \mathrm{m}$. Although cavitation is more intense in the smallest channel, jet impingement exposure surface is limited compared to the other configurations. Therefore, its thermal effects become less compared to those of the channel with diameter of $504 \mu \mathrm{m}$. The channel with diameter of $762 \mu \mathrm{m}$ delivers higher power output compared to the channel with diameter of 256 $\mu \mathrm{m}$. Although the jet impingement exposure surface is the largest for the channel with diameter of $762 \mu \mathrm{m}$, the transition in flow morphology results in a relatively lower power output.

To illustrate the potential of energy harvesting, the required power values for operation of some miniature electronic appliances are included in Table 1 . As can be seen, all of the

Table 1. Electrical Characteristics of Some Miniature DailyUsed Energy-Harvesting Devices ${ }^{27}$

\begin{tabular}{lcc}
\multicolumn{1}{c}{ device name } & $\begin{array}{c}\text { forward current } \\
(\mathrm{mA})\end{array}$ & $\begin{array}{c}\text { power dissipation } \\
(\mathrm{mW})\end{array}$ \\
light-emitting diodes (LEDs) & 80 & 176 \\
antenna GPS & 12 & 39.6 \\
digital mini thermometer & 300 & 450 \\
mini GPS receiver & 200 & 280 \\
portable charger for cell & 1000 & $1000-5000$ \\
phones & & \\
\hline
\end{tabular}

reported (generated) power values are within the range of the required power levels for these miniature appliances. Under the studied conditions, the proposed system is capable of powering approximately three light-emitting diodes and a digital mini thermometer. The reclaimed power values serve as the preliminary results and proof of concept for the proposed method. Necessary power for consumer devices, such as cell phones, laptops, and devices in offices, could be provided using this approach.

\section{CONCLUSIONS}

In this study, we showed that the exposure of small-scale cavitating jet flows exiting different micro-/minichannel configurations has the capability to increase the temperature on a thin solid surface, which can be used to exploit energy. The preliminary results show that the temperature rise ranges from 0.8 to $5.7{ }^{\circ} \mathrm{C}$ under the experimental conditions. To support the temporal results, a detailed set of visualization images was provided for different microtube diameters and different upstream pressures. Various morphological patterns exist for each case, and pattern shifts were also observed as the inner diameter of the microtube increased from 504 to $762 \mu \mathrm{m}$. The calculated obtainable power values can provide enough energy for many basic miniature electronic appliances, such as cell phones, laptops, and office devices. The only required input is a simple flow/injection of a cooling fluid, which could be well provided by miniature/embedded MEMS micropumps or recovery water lines from thermal fluid systems. Thus, this approach has the potential of meeting personal energy needs in an inexpensive and environment-friendly manner and offers considerable advantages compared to other energy-harvesting devices in terms of cost and energy implementation. Furthermore, the results could be further improved by optimizing the injection conditions, designing a functional solid surface having multiple cavitating jet arrangements, and integrating it to effective thermoelectric power generators.

\section{EXPERIMENTAL SECTION}

Experimental Setup. The cavitating-flow generation setup used in this study is shown in Scheme 1. An aluminum plate with a surface of $1 \times 1 \mathrm{~cm}^{2}$ and weight of $1.85 \mathrm{~g}$ is kept at a specific distance $(1.8 \mathrm{~cm})$ from the tip of the micro-/ minichannels to act as the exposed surface for impingement of the microjets resulting from the exiting spray. The depth of the aluminum plate utilized in this study was $1 \mathrm{~mm}$. Except certain cases, the area that was chosen to calculate the effect of temperature rise was $1 \times 1 \mathrm{~mm}^{2}$. In other cases, such as the microtube with diameter $152 \mu \mathrm{m}$ (which had a smaller impact area), modifications in calculations were considered to ensure a reasonable judgment. The tests are performed in four different channel configurations with diameters of $152,256,504$, and $762 \mu \mathrm{m}$, respectively. In the presence of intense cavitating flows downstream of the cavitating-flow generator device (micro-/ minichannel acts as a restrictive element connected to the stainless steel tube), a heating effect can be accomplished. The temperature variations are recorded using a thermal camera system, whereas the cavitating flows are visualized using a highspeed camera system.

Device Characterization. The setup generates cavitating flows with flow-restrictive elements, which are microchannels with different diameters. The stainless steel tubing with diameter of $4.5 \mathrm{~mm}$ is connected to these polyether ether ketone (PEEK) micro-/minichannels. The connection between the tubes is accomplished with proper Swagelok fittings to have a sudden reduction in diameter, thereby reducing the static pressure, which leads to the generation of cavitation bubbles and cavitating jet flows. Furthermore, the lengths of the PEEK channels are adjusted in such a way that the generated bubbles and vapor phase could reach the exit of the channels. Thus, this length is kept at $4.5 \mathrm{~mm}$ to better exploit the energy released from the collapse of the cavitation bubbles. To reduce light reflection from the aluminum plate and increase emissivity, 
Scheme 1. Schematic of the Proposed System for Generating Cavitation Bubbles and Micro/Mini Cavitating Jets ${ }^{a}$

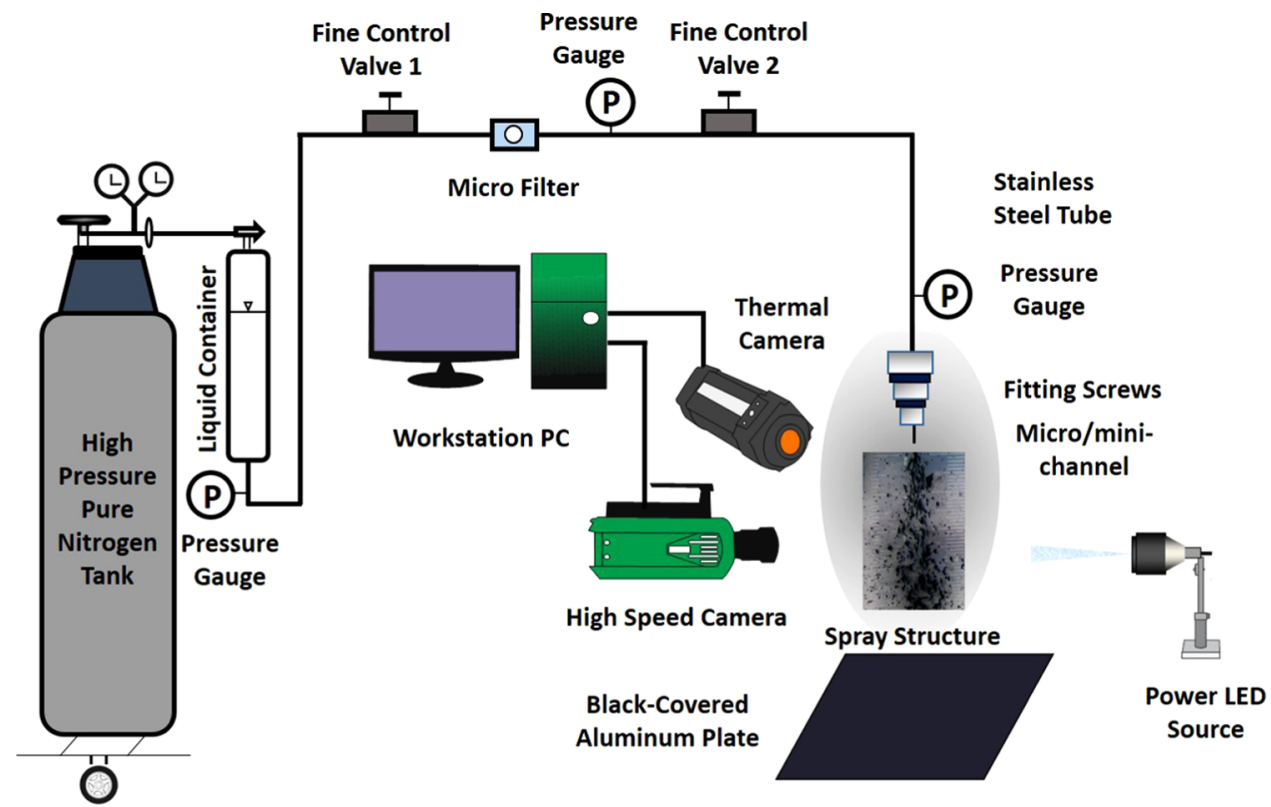

${ }^{a}$ A high-pressure pure nitrogen tank (Linde Gas, Gebze, Kocaeli, Turkey) supplies the required upstream pressure for the system. This tank is connected to a 1 gal fluid reservoir (Swagelok, Erbusco BS, Italy), which is filled with deionized water and serves as the working fluid. The reservoir is connected to the system with adapter fittings. Two pressure sensors (Omega) are mounted at the entrance and end of the tubing system to measure the pressures. Two fine control valves (Swagelok) are integrated to the system to control the flow at the desired locations. A micro T-type filter (Swagelok) with nominal pore size of $15 \mu \mathrm{m}$ is used to filter any particles larger than $15 \mu \mathrm{m}$.

besides the calibration of the thermal camera, the plate is colored black.

Experimental Procedure. The experiments are carried out in two separate steps. In the first step, temperature variations on the aluminum plate are measured with the aid of the thermal camera system. This plate is connected to the system via sustaining clamps, which hold it against vibration. A thermal camera (FLIR Systems) and a workstation with image postprocessing software capture the temperature variations on the aluminum plate that result from its exposure to the cavitating jet flows. The plate is held by means of suitable clamps at a distance of $1.8 \mathrm{~cm}$ from the exit of the channel. The selected upstream pressures are 10, 40, and 60 bar. The exiting jet is exposed to the atmospheric pressure of 1 bar. Surface temperatures are recorded at three different time steps for each micro-/minitube and each upstream pressure. Time steps are selected as 0 (just after), 30, and $120 \mathrm{~s}$ after the exposure of the surface to the cavitating flow.

In the second step, the flow structure is visualized utilizing a high-speed camera system for the aforementioned channels at different segments relative to the exit of the microchannels and injection pressures. The spray structure is divided into six identical segments from the emergence of the jet at the outlet of the channel down to the location where the cavitating jet interacts with the aluminum plate. Each segment has a length of $3 \mathrm{~mm}$. The visualization experiments are performed at several upstream pressures ranging from 5 to 60 bar. To study the spray formation in the different segments, the exposure time is adjusted to a very low value of $1 \mu \mathrm{s}$. The images of the cavitating flows are collected by a double-shutter CMOS camera, which allows the acquisition of two successive images with a resolution of $1280 \times 800$ pixels (pixel size of $0.02 \mathrm{~mm}$ ) within a very short time delay. The CMOS camera is equipped with a macro camera lens (type K2 DistaMax with focal length: $s=50 \mathrm{~mm}$ and $f$-number: $f=1.2)$ and is mounted at a distance of $342 \mathrm{~mm}$ from the imaging plane, yielding a magnification of $M=0.137$. This optical arrangement ensures that only the central region of the lens is used, where aberration can be neglected. The images are exposed in a background illumination mode using a pulsed LED array consisting of 551 high-performance LEDs with a total area of $160 \mathrm{~mm} \times 100$ $\mathrm{mm}$. In front of the LED array, an opaque plate is installed to produce diffuse illumination. The typical duration of the light pulses is $0.05-0.07 \mathrm{~ms}$, whereas the time delay between the two successive images is adjusted to the local flow velocity in the range of $1-3 \mathrm{~ms}$.

Flow Characterization. The mass flow rate is obtained by measuring the fluid mass passing from the outlet cross-sectional area over a given time period. The experiments are carried out at a broad range of upstream pressures, and all temperature measurements and visualization tests are repeated several times to ensure the reliability of the obtained results. Using the manufacturer's specification sheets and also the propagation from the uncertainty propagation method by Kline and McClintock, ${ }^{28}$ the average uncertainties in cavitation number $( \pm 6.7 \%)$, flow rate $( \pm 1.4 \%)$, inner diameter $( \pm 0.002 \mathrm{~mm})$, and pressure drop $( \pm 0.3 \%)$ are obtained.

\section{ASSOCIATED CONTENT}

\section{S Supporting Information}

The Supporting Information is available free of charge on the ACS Publications website at DOI: 10.1021/acsomega.7b01204.

Flow patterns at the outlet of the micro-/minichannels for upstream pressures of 10 bar (Figure S1); flow patterns at the outlet of the micro-/minichannels for upstream pressures of 40 bar (Figure S2); flow patterns at the outlet of the micro-/minichannels for upstream pressures of 60 bar (Figure S3); thermal contours 
captured by the thermal camera on the solid surface for different cases just after the exposure (Figure S4); thermal contours captured by the thermal camera on the solid surface for different cases $30 \mathrm{~s}$ after the exposure (Figure S5); and uncertainties in experimental parameters (Table S1) (PDF)

\section{AUTHOR INFORMATION}

\section{Corresponding Author}

*E-mail: kosara@sabanciuniv.edu.

ORCID $\odot$

Ali Koşar: 0000-0001-6283-6717

Notes

The authors declare no competing financial interest.

\section{ACKNOWLEDGMENTS}

The equipment and characterization support provided by the Sabanc1 University Nanotechnology Research and Applications Center (SUNUM) is appreciated. This work was supported by the Science Academy Outstanding Young Investigator Support Program (BAGEP) and the Turkish Academy of Science (TUBA) Outstanding Young Investigator Support Program (GEBIP).

\section{REFERENCES}

(1) Agarwal, A. K.; Som, S.; Shukla, P. C.; Goyal, H.; Longman, D. In-nozzle flow and spray characteristics for mineral diesel, Karanja, and Jatropha biodiesels. Appl. Energy 2015, 156, 138-148.

(2) Arndt, R. E. Cavitation in fluid machinery and hydraulic structures. Annu. Rev. Fluid Mech. 1981, 13, 273-326.

(3) Cioncolini, A.; Scenini, F.; Duff, J.; Szolcek, M.; Curioni, M. Choked cavitation in micro-orifices: An experimental study. Exp. Therm. Fluid Sci. 2016, 74, 49-57.

(4) Mohan, B.; Yang, W.; Yu, W. Effect of internal nozzle flow and thermo-physical properties on spray characteristics of methyl esters. Appl. Energy 2014, 129, 123-134.

(5) Payri, R.; Salvador, F.; Gimeno, J.; de la Morena, J. Study of cavitation phenomena based on a technique for visualizing bubbles in a liquid pressurized chamber. Int. J. Heat Fluid Flow 2009, 30, 768-777.

(6) Bourne, N.; Field, J. Shock-induced collapse of single cavities in liquids. J. Fluid Mech. 1992, 244, 225-240.

(7) Brennen, C. E. Cavitation and Bubble Dynamics; Cambridge University Press, 2013.

(8) Flannigan, D. J.; Suslick, K. S. Inertially confined plasma in an imploding bubble. Nat. Phys. 2010, 6, 598-601.

(9) Flannigan, D. J.; Suslick, K. S. Plasma formation and temperature measurement during single-bubble cavitation. Nature 2005, 434, 5255.

(10) Bostanci, H.; Rini, D. P.; Kizito, J. P.; Singh, V.; Seal, S.; Chow, L. C. High heat flux spray cooling with ammonia: Investigation of enhanced surfaces for HTC. Int. J. Heat Mass Transfer 2014, 75, 718725 .

(11) Hou, Y.; Tao, Y.; Huai, X. The effects of micro-structured surfaces on multi-nozzle spray cooling. Appl. Therm. Eng. 2014, 62, $613-621$.

(12) Wang, J.-X.; Li, Y.-Z.; Zhang, H.-S.; Wang, S.-N.; Mao, Y.-F.; Zhang, Y.-N.; Liang, Y.-H. Investigation of a spray cooling system with two nozzles for space application. Appl. Therm. Eng. 2015, 89, 115124.

(13) Ghorbani, M.; Yildiz, M.; Gozuacik, D.; Kosar, A. Cavitating nozzle flows in micro-and minichannels under the effect of turbulence. J. Mech. Sci. Technol. 2016, 30, 2565-2581.

(14) Skyba, D. M.; Kaul, S. Advances in microbubble technology. Coron. Artery Dis. 2000, 11, 211-219.
(15) Rapoport, N.; Gao, Z.; Kennedy, A. Multifunctional nanoparticles for combining ultrasonic tumor imaging and targeted chemotherapy. JNCI, J. Natl. Cancer Inst. 2007, 99, 1095-1106.

(16) Gañán-Calvo, A. M.; Gordillo, J. M. Perfectly monodisperse microbubbling by capillary flow focusing. Phys. Rev. Lett. 2001, 87, No. 274501.

(17) Cheng, W.-L.; Zhang, W.-W.; Chen, H.; Hu, L. Spray cooling and flash evaporation cooling: The current development and application. Renewable Sustainable Energy Rev. 2016, 55, 614-628.

(18) Suslick, K. S.; Flannigan, D. J. Inside a collapsing bubble: sonoluminescence and the conditions during cavitation. Annu. Rev. Phys. Chem. 2008, 59, 659-683.

(19) Zhou, J.; Wei, C.; Hu, J. A novel approach for predicting thermal effects of gas cavitation in hydraulic circuits. Energy 2015, 83, 576582.

(20) Bowen, C.; Kim, H.; Weaver, P.; Dunn, S. Piezoelectric and ferroelectric materials and structures for energy harvesting applications. Energy Environ. Sci. 2014, 7, 25-44.

(21) Caliò, R.; Rongala, U.; Camboni, D.; Milazzo, M.; Stefanini, C.; de Petris, G.; Oddo, C. Piezoelectric energy harvesting solutions. Sensors 2014, 14, 4755-4790.

(22) Arnaud, A.; Boughaleb, J.; Monfray, S.; Boeuf, F.; Cugat, O.; Skotnicki, T. Thermo-mechanical efficiency of the bimetallic strip heat engine at the macro-scale and micro-scale. J. Micromech. Microeng. 2015, 25, No. 104003.

(23) Ravindran, S. K. T.; Kroener, M.; Shabanian, A.; Goldschmidtboeing, F.; Woias, P. Analysis of a bimetallic micro heat engine for energy harvesting. Smart Mater. Struct. 2014, 23, No. 035011.

(24) Ghorbani, M.; Alcan, G.; Unel, M.; Gozuacik, D.; Ekici, S.; Uvet, H.; Sabanovic, A.; Kosar, A. Visualization of microscale cavitating flow regimes via particle shadow sizing imaging and vision based estimation of the cone angle. Exp. Therm. Fluid Sci. 2016, 78, 322-333.

(25) Sou, A.; Hosokawa, S.; Tomiyama, A. Effects of cavitation in a nozzle on liquid jet atomization. Int. J. Heat Mass Transfer 2007, 50, $3575-3582$.

(26) Ramadass, Y. Maximizing the Output Power from Thermoelectric Harvesters. Wireless Des. Dev. 2014, 22, 24-27.

(27) Cikim, T.; Gozuacik, D.; Kosar, A. Power Reclamation Efficiency of a Miniature Energy Harvesting Device using External Fluid Flows. Int. J. Energy Res. 2014, 38, 1318-1330.

(28) Kline, S. J.; McClintock, F. Describing uncertainties in singlesample experiments. Mech. Eng. 1953, 75, 3-8. 